\title{
Implementation of Multi-hop Routing Protocols for the Dramatic Range Enhancement of Wireless Sensor Networks
}

\author{
Vasileios Lakafosis*, and Manos M. Tentzeris \\ School of ECE, Georgia Institute of Technology, Atlanta, GA 30332-0250, USA \\ E-mail: vasilis@ece.gatech.edu
}

\begin{abstract}
In this paper, both general and routing-specific constraints and requirements that have to be satisfied during the design of wireless sensor networks are presented. The need for emergence of efficient protocols, especially those related to network layer, is stressed. A classification of the state-of-the-art routing techniques is also presented along with a benchmarking evaluation in terms of power requirements and range enhancement for "rugged" wireless sensor networks.
\end{abstract}

\section{INTRODUCTION}

Wireless Sensor Networks (WSNs) are composed of a large number of sensor nodes that, besides processing and communication capabilities, possess one or more sensing devices and, thus, are densely deployed either inside a physical phenomenon or very close to it.

The potential gains of being able to monitor and control the physical environment remotely can be exploited only if the design of the WSNs manages to address the numerous unique challenges posed. First of all, the sensor nodes carry limited, generally irreplaceable power sources and as a result the lifetime of a sensor network depends on the lifetime of the power units of the nodes, the drain of which is mainly caused by the data communication, including both transmission and reception, out of all the three domains involved with power consumption, namely sensing, communication, and data processing [3]. Secondly, although smaller and more powerful processors are being made available, the sensor nodes are still tightly constrained in terms of computational power and storage capacity. Another major factor influencing the design of the WSNs is the transmission media. The nodes comprising an "ad-hoc" wireless sensor network are interconnected with wireless links, which are almost always formed by radio, offer limited range of a few meters and bandwidth on the order of a few hundred kbps and, of course, face the traditional problems associated with a wireless channel such as fading, shadowing, reflection, scattering, diffraction and multipath. The carrier frequency for WSN transceivers is limited, because of certain hardware constraints and the tradeoff between antenna efficiency and power consumption, to the Ultra High Frequency (UHF) range, with the ISM (Industrial, Scientific and Medical) bands being a good choice, since they offer free radio, huge spectrum allocation and global availability but also potential harmful interference from existing applications [3]. Additionally, the sensor nodes have to be adaptive to the environment of the limited physical area they are observing, meaning they should be able to work unattended under perhaps extreme heat or cold, under high air or water pressure or in an extremely noisy environment. In addition to the above constraints, wireless sensor nodes may need to fit into small-sized modules, comparable to a matchbox. Finally, they are also required to have low production cost (and even be dispensable), so as to keep the overall cost of the WSN projects within reasonable bounds, although maintaining in the same time a large amount of functionalities. 


\section{Multi-Hop COMMUNICATION}

Recent advances in wireless communications and digital electronics have rendered the construction of relatively low-cost, low-power, multifunctional sensor nodes feasible. However, the deployment of large scale WSN infrastructures based on the collaboration of a large number of nodes has only become a reality through the embedded software implementing the different layers of the protocol stack developed the last years.

Specifically, since a generally large number of sensor nodes are densely deployed in WSN fields, multi-hop communication is exploited in the interconnection between nodes. First, since the transmission power of a wireless radio is proportional to the square of the distance or an even higher-order due to the close proximity of the antennas of the sensor nodes to the ground, multi-hopping both leads to less power consumption and lower cost than the traditional single hop communication and allows high spatial frequency reuse. Furthermore, multi-hop routing can effectively overcome shadowing and path loss effects offering coverage over large geographical regions. Improved sensing accuracy by distributed processing of large quantities of sensing information is also feasible through multi-hop communication [4]. Finally, the ability to sustain sensor network functionalities without any interruption due to sensor node failures can also be achieved because of the multiple paths available for the data to flow offered by multi-hop routing.

\section{NeTwORK ROUTING PROTOCOLS}

In addition to the general constraints mentioned in section I, network routing protocols designed from scratch are required to also meet some extra requirements posed by the WSN paradigm. First of all, hundreds to several thousands of nodes are deployed throughout the sensor field, with the node densities being as high as 20 nodes per square meter, and, thus, it is impossible to build a global addressing scheme as the overhead of the identification maintenance would be high; classical IP-based protocols are, therefore, rendered inapplicable to sensor networks [3]. Second, the deployment of the sensor nodes is usually random, meaning not pre-determined, and as a consequence the reduction of the installation cost, the elimination of the need for any pre-organization and re-planning, as well as the confrontation of frequent, and perhaps significant, topological changes because of node failures or blockages due to power outage, physical damage or environmental interference can be achieved only through the emergence of efficient self-organizing protocols. Furthermore, the data traffic generated by the sensor nodes has high spatial and temporal correlation since multiple sensors may generate same data within the vicinity of a sensed phenomenon and such redundancy needs to be exploited by the routing protocols to improve energy and bandwidth utilization. Specifically, recognizing that computation is far less power consuming than communication, tactics special to WSNs, such as in-network processing, clustering and data aggregation, namely the combination of data from different sources by using functions such as elimination of duplicates, can effectively cope with the aforementioned challenge. Fourth, time-constrained applications deem it necessary for the data delivery to be accomplished within a certain period of time or it will be useless. Last but not least, the mobility of a number of nodes may need to be supported, in which case the task of routing data from or to moving nodes becomes more challenging.

The WSN routing protocols can be classified into proactive, reactive and hybrid, depending on how a path is found between the source and the destination. In proactive protocols all routes are computed beforehand, while in reactive protocols routes are 
computed on demand. Hybrid protocols are based on a combination of these two approaches [2].

Another classification of the WSN routing protocols can be done into four main categories, namely data-centric or flat-architecture, hierarchical or cluster-based, location-based and Quality-of-Service based [1]. Protocols, which name the data and gather the sensed information based on some properties of the data rather than querying an individual node, are categorized as data-centric. On one hand, data aggregation can make efficient use of the WSN resources and the overhead of forming clusters or the use of specialized nodes can be avoided, but on the other hand complex queries might be impossible to be answered as well as the naming schemes are usually application-dependent. In hierarchical routing methods sensor nodes form clusters where the low energy nodes are used to perform the sensing in the proximity of the phenomenon, while the less energy-constrained nodes play the role of cluster-heads and process, aggregate and forward the information to a potential layer of clusters among themselves toward the base station. Another class of routing protocols is based on the location information of the sensor nodes either estimated on the basis of incoming signal strengths or obtained by small low-power GPS receivers or even by combination of the two previous methods. Location-based protocols use this information to reduce the latency and energy consumption of the sensor network. Finally, while setting up the paths in the WSN, QoS-based routing protocols have to balance between power consumption and satisfaction of certain QoS metrics, such as end-to-end delay and bandwidth when delivering data to the base station. Some of the most representative protocols applying the different principles of the four categories are shown and compared at the following table [2].

\begin{tabular}{|c|c|c|c|c|c|c|c|c|}
\hline & & $\begin{array}{l}\text { Power } \\
\text { usage }\end{array}$ & Scalability & $\begin{array}{l}\text { Multi- } \\
\text { path }\end{array}$ & $\begin{array}{l}\text { Query- } \\
\text { based }\end{array}$ & $\begin{array}{c}\text { Data } \\
\text { Aggregation }\end{array}$ & $\begin{array}{l}\text { Local- } \\
\text { ization }\end{array}$ & Mobility \\
\hline \multirow{9}{*}{ 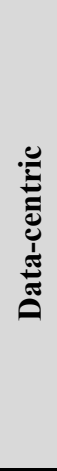 } & SPIN & limited & limited & yes & yes & yes & no & possible \\
\hline & $\begin{array}{c}\text { Directed } \\
\text { Diffusion }\end{array}$ & limited & limited & yes & yes & yes & yes & limited \\
\hline & EAR & N/A & limited & no & yes & no & & limited \\
\hline & $\begin{array}{l}\text { Rumor } \\
\text { Routing }\end{array}$ & N/A & good & no & yes & yes & no & $\begin{array}{c}\text { very } \\
\text { limited }\end{array}$ \\
\hline & GBR & N/A & limited & no & yes & yes & no & limited \\
\hline & CADR & limited & limited & no & no & yes & no & no \\
\hline & COUGAR & limited & limited & no & yes & yes & no & no \\
\hline & ACQUIRE & N/A & limited & no & yes & yes & no & limited \\
\hline & MCFA & N/A & good & no & no & no & no & no \\
\hline \multirow{5}{*}{  } & LEACH & maximum & good & no & no & yes & yes & $\begin{array}{c}\text { fixed } \\
\text { BS }\end{array}$ \\
\hline & PEGASIS & maximum & good & no & no & no & yes & $\begin{array}{c}\text { fixed } \\
\text { BS }\end{array}$ \\
\hline & $\begin{array}{c}\text { TEEN \& } \\
\text { APTEEN }\end{array}$ & maximum & good & no & no & yes & yes & $\begin{array}{c}\text { fixed } \\
\text { BS }\end{array}$ \\
\hline & SOP & N/A & low & no & no & no & no & no \\
\hline & VGA & N/A & good & yes & no & yes & yes & no \\
\hline \multirow{2}{*}{ } & GAF & limited & good & no & no & no & no & limited \\
\hline & GEAR & limited & limited & no & no & no & no & limited \\
\hline
\end{tabular}




\begin{tabular}{|c|c|c|c|c|c|c|c|c|}
\hline \multirow{2}{*}{} & SAR & N/A & limited & no & yes & yes & no & no \\
\cline { 2 - 8 } & SPEED & N/A & limited & no & yes & no & no & no \\
\hline
\end{tabular}

\section{FUTURE WORK}

The most important representatives of each of the aforementioned categories of WSN routing protocols are to be implemented and deployed with the use of a relevantly large number of low power, low cost, miniaturized wireless sensor motes. The performance of the routing in each case is to be investigated under the different propagation effects caused by the three different testbed frequency bands, namely the ISM $433 \mathrm{MHz}, 915 \mathrm{MHz}$ and $2.4 \mathrm{GHz}$, as well as with different typical RFID antennas. Preliminary results of the power lifetime efficiency and the range improvement achieved by the protocols, as well as the tradeoffs between these two factors mainly in open-space environments will be obtained.

\section{REFERENCES}

[1] K. Akkaya, M. Younis, "A Survey On Routing Protocols For Wireless Sensor Networks", Ad Hoc Networks, pp. 26, 2003.

[2] J. N. Al-Karaki, A. E.Kamal, "Routing techniques in wireless sensor networks: a survey", Wireless Communications IEEE, vol. 11, no. 6, pp. 6-28, December 2004.

[3] I. F. Akyildiz, W. Su, Y. Sankarasubramaniam, E. Cayirci, "Wireless sensor networks: a survey", Computer Networks (Elsevier) Journal, vol. 38, no. 4, pp. 393-422, March 2002.

[4] W. R. Heinzelman, J. Kulik, H. Balakrishnan, "Adaptive protocols for information dissemination in wireless sensor networks", Proceedings of the ACM MobiCom'99, pp. 174-185, 1999. 